\title{
Bayesian updating for uncertain condition state using permanent monitoring and sequential inspections
}

\author{
Christelle Geara ${ }^{1,2^{*}}$, Rafic Faddoul $^{1}$, Alaa Chateauneuf ${ }^{2,3}$ and Wassim Raphä̈ ${ }^{1}$ \\ ${ }^{1}$ ESIB, Université Saint-Joseph, Mar Roukos, PoB. 11-154, Riad El Solh, Beyrouth, Liban \\ ${ }^{2}$ Université Clermont Auvergne, CNRS, Institut Pascal, 63000 Clermont-Ferrand, France \\ ${ }^{3}$ CIDECO, 2 av. Blaise Pascal, 63178 Aubière, France
}

\begin{abstract}
Structural Health Monitoring using permanent sensors has been a fast growing management tool during the last decade. However, till this date, the installation of sensors on every measurable feature of the structure stills prohibitory costly. In this paper, an approach is proposed for a Bayesian updating of the condition states, based on the output of the monitoring sensors. The posterior probability distribution describing the magnitude of the defect for each element is further updated by inspecting some elements in an optimal sequence. A numerical application of the proposed methodology is presented for a concrete frame structure.
\end{abstract}

\section{Introduction}

Managing the lifetime of structures has been a challenge especially that we are facing an accumulation of maintenance needs due to the lack of budget. Thus, the fast progress of Structural Health Monitoring (SHM) resulted in decreasing costs and improving efficiency. Two main approaches can be adopted in SHM: (i) the local technique providing direct evaluation of a structural member to assess its state, and (ii) the global technique where optimally installed sensors allows monitoring the whole structure [1]. While the former might give more precise results, it can't be applicable for a complex structure, nor on non-accessible structural members. In such cases, it is preferable to monitor the structure indirectly using the global SHM approaches relying on a mechanical modeling of the structure.

Conventional inspection technologies going from visual to destructive and/or nondestructive techniques are being widely used. These techniques can be fruitful in some situations yet, results are only given at discrete time points which might keep some critical defects undetected for a period of time, if they occurred between two successive inspections.

In this paper, we introduce a methodology relying on a Bayesian update of the condition state of the structure to find an optimal maintenance planning using information from various data sources. Section 2 describes the proposed methodology and Section 3 illustrates the applicability of the method by a numerical application.

*christelle.geara@net.usj.edu.lb 


\section{Methodology}

The proposed methodology seeks to obtain an optimal planning using information from permanent SHM and sequential inspections which will reduce the uncertainty related to the condition state of the whole structure. Specifically, the posterior Bayesian probability distributions describing the magnitude of the defect for each element are further updated by inspecting only a few elements in an optimal sequence. We assume the structural response is obtained by measuring modal parameters and a structural damage is defined by a loss in the stiffness of the structural elements. The steps of the proposed methodology are as follows:

1) Obtain the a priori information about the degradation extent and the data from SHM;

2) For each element, calculate the a posteriori distribution of the degradation extent;

3) According to the results in step 2, optimally decide if an inspection is needed and on which element. If no inspection is required, go to step 6;

4) If an inspection is done on a particular element, its results are used to compute a new a posteriori distribution for each element of the structure;

5) Steps 3 and 4 are repeated until the optimal action consists on performing maintenance actions on the element instead of the inspection;

6) Optimally choose a maintenance action for each element of the structure.

The $a$ posteriori distribution is computed by a Bayesian update $[2,3]$ which involves all the uncertainties affecting the assessment of the structural system. In our case, we use the Approximate Bayesian Computation algorithm [4] to calculate the a posteriori distribution of the structural parameters using the Modal Assurance Criterion matrix defined by :

$$
M A C_{i, i}=\frac{\left[\Phi_{i}^{T} \Phi_{i}\right]^{2}}{\left[\Phi_{i}^{T} \Phi_{i}\right]\left[\Phi_{i}^{T} \Phi_{i}\right]}
$$

where i corresponds to the vibration mode and $\Phi_{i}$ and $\Phi^{\prime}{ }_{i}$ are the eigenvectors.

The final step is to optimally decide whether to make further inspections and/or maintenance actions [5]. Let's consider $\theta \in \Theta=\{1,2, \ldots 1\}$ a set of possible states of the nature describing the degradation extent of an element (e) and $(r)$ the result of an inspection. The $a$ posteriori probability distribution of the condition state of (e) is updated as follows[6]:

$$
P\left[\theta^{e}=s \mid r\right]=\frac{P\left[r \mid \theta^{e}=s\right] * P\left[\theta^{e}=s\right]}{\sum_{i=1}^{l} P\left[r \mid \theta^{e}=i\right] * P\left[\theta^{e}=i\right]}
$$

\section{Numerical application}

We consider the case of a concrete frame structure composed of $20 \times 60 \mathrm{~cm}$ vertical and $20 \times 40 \mathrm{~cm}$ horizontal rectangular elements (figure 1). We assume that elements 1 and 2 are damaged, having lost $40 \%$ of their initial modulus (33GPa), but the damage has not been detected yet. A sensor is implemented on the node (N3) to detect damages.

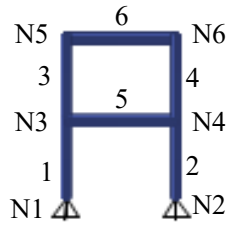

Fig. 1. Simply supported concrete frame 
The a priori distribution of the degradation of all the elements is supposed to be uniform non informative, the inspection is assumed to be perfect and the actions could either be nothing to do, the repair of the element or its replacement having respectively a cost of 0 monetary units (m.u.), 2 m.u. and 7 m.u. The continuous damage space $(0,1)$ of each element is discretized into four ordinal states $\bar{\theta}^{e}$ with $\alpha_{e}$ the percent of remaining stiffness as follows:

- $\quad \bar{\theta}^{e}=1$ with $\alpha_{e} \in[0,0.25]$ inducing a cost of 12 m.u. ;

- $\quad \bar{\theta}^{e}=2$ with $\alpha_{e} \in[0.25,0.5]$ inducing a cost of 9 m.u. ;

- $\quad \bar{\theta}^{e}=3$ with $\alpha_{e} \in[0.5,0.75]$ inducing a cost of 6 m.u. ;

- $\quad \bar{\theta}^{e}=4$ with $\alpha_{e} \in[0.75,1]$ inducing a cost of 1 m.u. ;

In our case, the optimal path consists of inspecting the element 1 and accordingly, applying maintenance actions on the remaining elements. The following table resumes the results by identifying, $\mathrm{P}\left(\bar{\theta}^{e}=\mathrm{i}\right)$ of element 1 and the actions to be done on all the elements. Abbreviations $N, R r$ and $R e$ stand respectively for "Nothing", "Repair" and "Replace".

Table 1. Summary of the optimal actions to be done on each element.

\begin{tabular}{|c|c|c|c|c|c|c|c|}
\hline \multirow{2}{*}{$\bar{\theta}^{e}$} & \multirow{2}{*}{$\mathrm{P}\left(\bar{\theta}^{e}\right)$} & \multicolumn{6}{|c|}{ Optimal actions on elements } \\
\cline { 3 - 8 } & & 1 & 2 & 3 & 4 & 5 & 6 \\
\hline 1 & 0.02 & $\mathrm{Re}$ & $\mathrm{Rr}$ & $\mathrm{N}$ & $\mathrm{N}$ & $\mathrm{N}$ & $\mathrm{N}$ \\
\hline 2 & 0.46 & $\mathrm{Re}$ & $\mathrm{Rr}$ & $\mathrm{N}$ & $\mathrm{N}$ & $\mathrm{N}$ & $\mathrm{N}$ \\
\hline 3 & 0.47 & $\mathrm{Rr}$ & $\mathrm{Rr}$ & $\mathrm{N}$ & $\mathrm{N}$ & $\mathrm{N}$ & $\mathrm{N}$ \\
\hline 4 & 0.05 & $\mathrm{~N}$ & $\mathrm{Rr}$ & $\mathrm{N}$ & $\mathrm{N}$ & $\mathrm{N}$ & $\mathrm{N}$ \\
\hline
\end{tabular}

Results showed that element 1 is probably between states 2 and 3 . These results meet our expectation since element 1 is assumed to have lost $40 \%$ of its initial modulus (unknown in a real problem) which corresponds to the upper limit of the state 2. Furthermore, the optimal actions to be done on each element looks to be very accurate since elements 1 and 2 are the only ones damaged. Hence, they should be the ones to be replaced or repaired while the other ones are in a good condition. We can notice also that as we move from a state to a better one, the actions are becoming less severe and less costly which means that there is a good correlation between the state of the element and the action to be done on it.

\section{Conclusion}

In this paper, an approach is proposed for a Bayesian updating of the condition states of all the structural elements, based on the output of the monitoring sensors and the results of the chosen inspections. It provides the decision maker with the opportunity to decide judiciously which element(s) need to be inspected by applying a decision analysis for the elements in the structure. It also takes into consideration the difficulty of access of each element and reduces the uncertainty of the results by mixing information from different sources.

\section{References}

1. G. Boscato, A. Dal Cin, S. Russo, F. Sciarretta, Adv. Mater. Res. 838, 2071-2078 (2014)

2. M.W. Vanik, J.L. Beck, S.K. Au, J. Eng. Mech. 126, 7 (2000)

3. K.V. Yuen, S.C. Kuok, Earthq. Eng. Struct. Dyn. 44, 757-774 (2015)

4. R. Faddoul, W. Raphael, A. Chateauneuf, Struct. Infrastruct. E. 7, 783-796 (2011)

5. P. Mimović, J. Stanković, V. Janković Milić, Ekon. Istraz. 28, 868-878 (2015) 